\title{
ESTRUCTURACION DE CLASES Y DESIGUALDAD: LA ESPAÑA DE LOS AÑOS 70 Y 80
}

\author{
Faustino Miguélez Lobo \\ (Universitat Autònoma de Barcelona)
}

\section{EL PROBLEMA PLANTEADO}

Los últimos 15 o 20 años han registrado cambios profundos y acele. rados en la estructura social española. El nivel más llamativo de cambio tiene su base en el terreno político y se refiere a las posibilidades de ex. presión y estructuración de intereses que gran parte de la sociedad no tenía a principios de los 70 . Se trata de un proceso de normalización que legitima y da carta de ciudadanía a la lucha por los intereses particularistas, a la competitividad por ascender socialmente. En teoxía se compite en igualdad de oportunidades, pero tanto la situación laboral como el estatus familiar y otros factores desequilibran en la práctica tal igualdad teótica.

Los cambios han sido también muy profundos en el trabajo, a consecuencia de la crisis y la reestructuración. Sería limitado centrarse aquí en los cambios meramente materiales - que, por supuesto, son importantesolvidando el análisis de las estrategias de los diversos sujetos implicados. La crisis es un elemento clave, desde el punto de vista del análisis sociológico, por cuanto se presenta en un momento en que personas, grupos y fuerzas sociales pueden empezar a contar con formas normales de expresión que hasta entonces no habían tenido legaimente: partidos, sindicatos, instrumentos de presión de diverso tipo, medios de comunicación, canales de movilidad, etc.

Hay un tercer nivel en el que la investigación parece más difícil, pero en el que los cambios son de trascendencia indudable para el problema 
que nos ocupa. Me refiero a cambios en los valores y en las pautas de comportamiento. Se ha hablado de crisis de valores colectivos como solidaridad, organización colectiva y otros para dar pie a concepciones más individualistas y más centradas en las exigencias de la petsona.

La pregunta que nos planteamos es hasta qué punto estos cambios están incidiendo sobre la estructuración de clases en nuestro país. Es decir, en qué grado están cambiando las características que presentan las clases sociales, así como las formas en que se relacionan. Por supuesto, no son pensables cambios tevolucionarios ni la desaparición de factores tradicionalmente presentes en la estructuración de clases; por el contrario, los cambios mismos que dibujaremos son en parte resultado de estrategias sociales en presencia.

En concordancia con este estado de la cuestión, se plantean las hipótesis siguientes: que los cambios en la estructuración de clases introducen ciertos rasgos que podríamos calificar de «modernización» en el sentido de semejanza con otros países de nuestro entorno; que tales cambios tienen que ver con transformaciones profundas y duraderas en la situación en el trabajo y con pautas de comportamiento y valores ligados a cambios en los intereses tanto en el trabajo como en la política; que, como consecuencia de esos cambios en la estructuración de clases, aparecen formas nuevas de desigualdad que son la manifestación más llamativa de la nueva correlación social.

\section{TRANSFORMACIONES EN LA ESTRUCTURACION DE LAS CLASES}

En el breve espacio de un articulo no puedo proponer un análisis detallado de la estructuración de clases, sino más bien señalar los que son, a mi juicio, los aspectos hoy más significativos del cambio que está teniendo lugar. Quiero referime fundamentalmente a tres, sin que esto haya de equivaler a ninguna pretensión de exhaustividad: la fragmentación de clases, el ascenso de las nuevas capas medias, la emergencia de la ideología tecnocrática.

\section{La fragmentación de la estructura tradicional}

Los cambios en el trabajo y en la economía, también en las formas de pensar y en las formas de vida, parecen estar fragmentando la estructura 
de clases tradicional. Se ha señalado principalmente la fragmentación de la clase obrera, pot cuanto la propia situación en el trabajo provoca la existencia de sectores con dificultades de comunicación entre sí; ocupados frente a parados, estables frente a inestables, trabajadores a tiempo pleno frente a trabajadores a tiempo parcial, trabajadores legales o declarados frente a sumergidos o ilegales, pueden ser algunas de las manifestaciones de dicho fenómeno; o bien la situación laboral introduce diferencias importantes en los objetivos que persiguen los diversos colectivos (empleo, salario, condiciones de trabajo, promoción, más autonomía), poniendo el acento tespectivamente sobre unos u otros, según los grupos laborales de que se trate; o bien los instrumentos que se utilizan para lograrlos y legitimarse son diversos (sindicatos generales, de categoría o de empresa, rechazo de la sindicación y opción por formas personalistas de relación bien desde una posición de debilidad - trabajadores sumergidos, por ejemplo- bien des. de una posición de fuerza - técricos y profesionales).

Pero tal fragmentación se ha dado también en otros niveles de la estructura de clases tradicional. Por ejemplo, las nuevas capas medias que muestran claras diferencias con las capas medias tradicionales; o la apari. ción de nuevas elites sociales que luchan con las tradicionales por el control de los resortes fundamentales del poder económico y político, así como de la infuencia ideológica.

Pero el problema no es sólo un problemna teórico, es decir, de búsqueda de nuevos indicadores para esta nueva estructuración de clases, sino también de significado práctico de la fragmentación misma en la estructuración de las clases y en definitiva en el funcionamiento de la sociedad. Veamos algunas cuestiones al tespecto.

Las organizaciones sociales tradicionales se encuentran con una dificultad cada día mayor para agregar las demandas tan diversificadas de sus tradicionales representados y presentarlas en propuestas y plataformas únicas, esto es, aceptables globalmente por todos ellos. Esto explica la oposición tan radical de algunas de estas organizaciones a ciertos fenómenos que están en el origen de la fragmentación - por ejemplo, la oposición de los sindicatos al trabajo precario, a la flexibilización, etc.-, pero explica también las dificultades con las que una estrategia de recomposición puede chocar. No es casuatidad que algunos grandes sindicatos europeos empiecen a dar mucha importancia a estrategias externas al trabajo, dirigidas a lograr una distribución de los recursos públicos más favorable a los trabajadores con ingresos más bajos, no limitándose meramente a los recursos generados en el momento de la producción; ello sugiete la aparición de nuevas formas de articulación de intereses que abarcan a colectivos en posiciones diferenciadas con respecto al trabajo. 
Mientras tanto, lo que parece más evidente es la acentuación, en sentido localista y gremialista, de tendencias corporativistas existentes en los colectivos laboralmente más seguros y sus familias empujando, al mismo tiempo, a mucha gente hacia la disgregación y el anonimato; la situación de estos últimos no sería precisamente envidiable, puesto que serían pasto de grupos muy cerrados o de un Estado que, en esas circunstancias, setía más y más tecnocrático.

\section{El desarrollo de las nuevas capas medias}

Las transformaciones económicas, políticas y culturales han hecho aumentar notablemente el sector técnico y profesional, aquellos cuya ocupación está ligada a aspectos técnicos, organizativos y de control en las empresas, a la administración y al desarrollo de las diversas políticas públicas, a las actividades culturales, etc. Se trata de capas sociales desartolladas en estrecha telación con la ocupación -a veces en relación con «nuevas ocupaciones»--, pero en una situación de trabajo totalmente segura y con sólidas perspectivas de mejora - lo que los autores de la teoría dualista (Piore, 1983) o segmentalista (Edwards, Gordon, Reich, 1985) llaman el segmento primario independiente--, es decir, una ocupación que les da seguridad, promoción, poder e influencia en muchos casos.

De todos estos aspectos, su cercanía al poder, mayor o menor según los colectivos, es la característica dominante. Esta cercanía al poder permite a las capas altas controlar influencias, tener acceso privilegiado a información, influir en la elaboración de las leyes, lo que a menudo se traduce en la obtención de buenos negocios para ellos mismos o para sus familias.

Estos sectores producen, organizan o controlan una serie de bienes de gran importancia estratégica en nuestra sociedad. Su relación con una actividad va mucho más allá de la inserción en una empresa o en una institución con unos intereses concretos y unos objetivos específicos. Tampoco se trata sólo de que puedan gozar de unas condiciones de vida mejores que otros sectores porque perciben más ingresos, o de que puedan tener mejores condiciones laborales o de que gocen de especiales relaciones de trabajo. Se trata de que los recursos que tienen entre manos son algo que afecta al conjunto de la sociedad, configurando lo que se suele llamar la modernización de las relaciones Estado-sociedad. Aquí está una de las razones básicas de su fuerza e influencia y lo que les da, a ellos y a sus hijos, el acceso a posiciones de responsabilidad, privilegio y promoción.

Parte de los nuevos valores y pautas de comportamiento que se están 
desarrollando son coherentes con la rapidez y la importancia del ascenso social de estas nuevas capas medias. La competitividad individual es uno. de los ejes dominantes de las relaciones, contra otros valores más solidarios y colectivos en el pasado que estas mismas capas mantenían gracias al contacto con la clase trabajadora. El éxito individual, medido a través de la obtención de ingresos elevados y el estatus social alto, se convierte en un indicador de capacidad indiscutible y en una forma de legitimación en la sociedad. Por contra, la pobreza y la escasez de oportunidades tienden a ser vistas como indicios de poca valía personal. La razón estaría en que nuestra sociedad ya no niega sus derechos a muchos, como bajo la dictadura, sino que los reconoce a todos en una situación donde, teóricamente, la posición social tiende a tevalidarse en un mercado abierto, sobre la base de las capacidades que cada uno aporte (Giddens, 1979).

Pero no deja de haber contradicciones entre los intereses reales y esta. «nueva ideología»; se manifiestan tales contradicciones, por ejemplo, en la existencia de ciertos vínculos grupales internos que llevan a estas capas: a desarrollar fórmulas de autoprotección del grupo, a una utilización doméstica y de amiguismo de los tecursos públicos y a dar más importancia a estas relaciones y a la confianza personal que a las presuntas capacidades técnicas, negando así, en la práctica, lo que es su propia base de legitimación. (Miguélez, Solé 1987).

\section{La ideología tecnocrática}

Los acelerados cambios en la economía, la política y la cultuta, así como el pujante ascenso de las nuevas capas medias al que me he referido, están siendo el caldo de cultivo de la nueva ideología dominante que podríamos calificar de tecnocratismo para sintetizar algunos de sus rasgos fundamentales. Una ideología que, asumida en gran parte por estas nuevas. capas medias, sin embargo, responde mucho más a intereses, ambiciones. y simbolismos de las viejas y, sobre todo, de las nuevas elites.

Podemos referirnos a la racionalidad económica por encima de cualquiera otra racionalidad como al rasgo más evidente de dicha ideología, una racionalidad que no se asume sólo como propia de la empresa priva. $\mathrm{da}$, sino que ha invadido la pública, la administración, las instituciones de todo tipo, aun las culturales. La relación costo-beneficio, medida por el arbittaje presuntamente neutral del mercado, es el criterio de toda eficacia; tiende a ser defendido sobre todo lo demás, sea esto la participación de los ciudadanos y el seguimiento de ciertos ritmos de cambio, sean las condiciones de trabajo, sea el contenido del mismo servicios que se ofrece 
(por ejemplo, dar más importancia, en la práctica, a que TV no tenga pérdidas que al contenido de sus progtamas). Este tipo de racionalidad permite a las elites gobernantes escapar más fácilmente al control diario no sólo de la oposición, sino también de la opinión pública o de organizaciones sociales no acordes con la línea política que se mantiene.

Está luego el convencimiento de la centralidad del desarrollo científicotécnico que suele ir acompañada de un posicionamiento muy acrítico ante el mismo. Tal desarrollo es visto y presentado por la ideología tecnocrática dominante como una variable externa y casi mágica, que se cree por sí misma alentadora del progreso, se invoca para frenar ciertas reivindicaciones o para justificat casi cualquiet estrategia. Como extetna e intocable deja, una vez más, a quienes gobieman las empresas, las instituciones o el Estado, al amparo de curalquier intervención controladora por parte de la sociedad.

En tercer lugar hay que referirse a una concepción totalmente jerarquizada de la sociedad y de las instancias colectivas; no hay posibilidad de que las cosas funcionen sin esa jerarquización que, por lo demás, es presentada como correspondiente a la existencia de capacidades diferenciadas que es necesario recompensar diferenciadamente; o las capacidades o los votos han designado las personas que están en la cúspide y es mal admitida la crítica a las mismas - que tiende a ser presentada como ctítica a las formas de convivencia aceptadas - o se tienden a rechazar formas de participación estables siempre en atas a la presunta eficacia y a la presunta competitividad con otras organizaciones u ottos países. Pero una vez en la cumbre el tecnócrata se cree infalible e intocable: sus decisiones son las mejores, porque son aquellas que cuentan con más apoyo de conocimientos técnicos, sus errores son insalvables errores de la técnica. (McLennan 1989).

Las que podríamos llamar las «nuevas elites» son los principales transmisores de esta ideología - las nuevas elites manageriales y políticas, técnicas, comunicacionales y culturales, es decir, los sectores más altos de las «nuevas capas medias»- que se tevela no sólo eficaz para penetrat en áreas de poder e infuencia, sino también como barrera protectora de su posición en la cumbre, pues nadie en mejor posición que las elites para ir en cabeza de la innovación, para evaluar los logros o para valorar la eficacia de la gestión. Formadas en el curso de muy pocos años y en parte todavía inseguras de su poder e influencia, las nuevas elites tienden a autoprotegerse y a vincularse por múltiples lazos a pesar de las posibles diferencias políticas, peto, al mismo tiempo, a acercarse a la clase dominante tradicional.

La modernización rápida y a toda costa es la bandera que las nuevas 
elites proponen a la sociedad. Pero no parece que la participación ciudadana para definir el presente y el futuro, en toda su complejidad, haya aumentado mucho. Por ello la modernización la definen las elites hasta los mínimos detalles: qué tipo de reestructuraciones, qué tipo de gastos, qué tipo de consenso social y hasta, dentro de poco, qué tipo de organizaciones sociales van a ser viables y cuáles no. Así, tecnocratismo y elitismo pueden estar invadiendo la cotidianidad de las relaciones sociales (en la política y fuera de ella) de tal manera que esto sea uno de los factores que expliquen fenómenos tan dispares como el absentismo en la política por un lado y los brotes de protesta - a veces poco duraderos porque son difícilmente organizables, a veces estrambóticos- por otro.

\section{LA ESTRUCTURACION DE CLASES: UN EJEMPLO CONCRETO}

Sobre los datos de Ia Enquesta Metropolitana de Barcelona (Izquierdo, Miguélez, Subirats, 1986), vamos a descifrar algunos de los factores concretos que intervienen actualmente en la estructuración de clases. Conviene advertir que operamos con un indicador de clase limitado, la categoría socioeconómica, con el objetivo de poder apteciar la infuencia de tales factores; ciertos aspectos más directamente políticos y culturales no son captados con tal indicador. En la tabla adjunta señalamos una distribución en base a tres grandes bloques diferenciando, en el Área Metropolitana, la ciudad de Barcelona del testo del Area. ${ }^{1}$

Distribución de las categorías socioeconómicas, según lugar de residencia

\begin{tabular}{lcccc}
\hline & Barcelona & Resto áred & Total \\
\hline Categorías altas $\ldots \ldots \ldots \ldots \ldots \ldots \ldots \ldots \ldots \ldots$ & 20,5 & 5,7 & 14,5 \\
Categorías medias $\ldots \ldots \ldots \ldots \ldots \ldots \ldots \ldots \ldots$ & 37,8 & 23,2 & 31,9 \\
Categorías bajas $\ldots \ldots \ldots \ldots \ldots \ldots \ldots \ldots \ldots$ & 38,3 & 67,9 & 50,3 \\
No clasificables $\ldots \ldots \ldots \ldots \ldots \ldots \ldots \ldots \ldots \ldots$ & 3,4 & 3,2 & 3,3 \\
& $(2,928)$ & $(1.984)$ & $(4.912)$ \\
\hline
\end{tabular}

1. Las categorías se distribuyen en los tres bloques de la manera siguiente: en las categorías aitas están los empresarios con asalariados, los profesionales líberales, los directores-gerentes, los técnicos altos; en las categorías medias se ubican los empresarios 
Es en las categorias altas donde principalmente se ubican las nuevas capas medias más dinámicas, a las que nos referíamos en el apartado anterior $y$, por supuesto, las elites, nuevas $y$ antiguas. Si comparamos estos datos con los tesultantes del Censo de 1971 (o Padrón de 1975), con precaución, dado que en nuestro caso se trata de datos muestrales, podemos observar que la proporción de estas categorías aumenta, pero que sobre todo el aumento se da en la gran urbe (Barcelona) donde se van concentrando los centros decisionales - -económicos, sociales, políticos y culturales- - que son aquellos en que estas capas están presentes y donde, además, residen.

También han experimentado crecimiento las categorías medias, y en particular en ellas el que podríamos calificar como «sector bajo de las capas medias», si la expresión no resulta demasiado complicada, es decir, los técnicos medios. Igualmente, la diferencia entre la urbe central y la periferia es notable a favor de la primera, aunque no tan marcada como en el caso anterior. Por último, las categorias bajas han decrecido - a favor del bloque anterior, presumiblemente- $\mathrm{y}$ en este caso los residentes de la periferia casi dobian a quienes residen en el centro.

Por supuesto que centro y periferia han de ser tomados aquí con un cierto relativismo en su acepción geográfica, por cuanto ciertas zonas del «Resto del Area», como el Vallés Occidental, tienen una distribución categorial muy semejante a la parte utbanísticamente mejor equipada de la ciudad de Barcelona, mientras que otras zonas de la misma Barcelona, por ejemplo Nou Bartis o Ciutat Vella, se asemejan a zonas extremas del Átea.

En la distribución categorial intervienen factores tradicionales: ser asalariado o ser trabajador independiente, tener o no tener propiedades; pero cada vez incide más en el nivel de estudios que se ha adquirido -sobre todo en las «nuevas capas medias»- o el tipo de trabajo, sólido o precario, que se ocupa o bien de tener o no tener trabajo, o la diferencia entre ser activo o no serlo. Vamos a referimos en particular a los aspectos Iaborales.

sin asalariados y los autónomos, los técnicos medios, los administrativos, los contramaes. tres; en las categorías bajas, fnalmente, los obreros cualificados, los obreros no cualificados, los trabajadores de los servicios, los trabajadores agratios. 


\section{ESTRUCTURACION DE CLASES Y TRABAJO}

Poder trabajar o no se ha convertido en una importante línea divisoria en nuestra sociedad que bloquea del lado del no trabajo los proyectos de futuro, los objetivos de igualdad o de movilidad o las pautas de integración social activa para mucha gente. Las tasas de paro son, según nuestra encuesta, de 3 o 4 puntos superiores a lo que registran los datos oficiales, si asumimos una concepción subjetiva.del paro que se expresa no sólo en el deseo de trabajar, sino también en cierta búsqueda de trabajo, aunque no necesatiamente por los cauces reglamentados; la razón de esta interpretación más «laxa» del concepto de paro está en que puede haber mucha gente deseosa de trabajar pero que haya abandonado las formas de búsqueda reglamentadas, convencidos de que son infructuosas.

La situación de pato tiene hoy tres posibles alivios: las subvenciones del Estado, el trabajo ilegal, Ios recursos obtenidos por otros miembros de Ia familia. Respecto a la primera posibilidad, cabe señalar que el nivel de cobertura es muy bajo y que posibilita medias de ingresos muy cetcanas al salario mínimo. El trabajo sumergido, estrictamente como tal, afecta en el Area de Barcelona, según nuestros datos, a más del 11 por ciento de la población mayor de 18 años, principalmente mujeres y básicamente en los servicios descualificados (servicios personales), en el comercio y en el textil; es presumible que sus condiciones económicas no sean muy buenas y que tendetá a crecer. Por lo que toca al tema de la familia, la realidad es que ésta es la solución verdadeta para muchos; la proporción de parados no cabezas de familia es 3 veces superior a la de parados cabezas de familia, lo cual, al tiempo que nos señala que es la familia la que está aguantando los efectos de la reestructuración economica, abona la hipótesis de que la estructura jerárqutica de ésta se refuetza, subordinando aún más a algunos de sus miembros.

Desde el punto de vista de la comprensión de nuestra sociedad, el paro - sobre todo el de larga duración- es importante no sólo porque debilita el nivel de vida de muchas personas, sino porque las coloca en situación de ciudadanos, personas o familiares de segunda categoría. Justamente por esta razón, no todos tienen las mismas probabilidades de quedar en paro: el origen social, el nivel de estudios, la edad y el sexo son aspectos que condicionan. Tipológicamente, el candidato a parado de larga duración es petsona de origen social bajo, sobre todo si tiene pocos estudios, más si es mujer, bastante si es joven. El parado de corta duración tiene estudios medios, puede ser de origen social intermedio, en general es joven, más probablemente varón que mujer. 
También las transformaciones del trabajo en el período reciente juegan un papel importante en la dinámica de la estructuración social. Frente a quienes tienen un trabajo estable, está casi el 18 por ciento de personas con trabajo precario -además de los sumergidos a los que nos referíamos anteriormente- que trabajan principalmente en la construcción, en los servicios públicos y sociales, pero también en el textil y el comercio. Se trata de personas que trabajan temporalmente, a tiempo parcial, a horas (a veces con contratos de una hota diaria). Frente a la seguridad en el trabajo, estabilidad en los ingresos, posibilidades de defender esa estabilidad a través de los convenios y los sindicatos, posibilidades de promoción en algún grado, se sitúa la precariedad de no saber si el mes que viene se tendrá contrato y por tanto ninguna de las ventajas mencionadas. Se trata de una enorme desigualdad que, una vez más, sería limitativo en exceso medir en términos económicos, cuando la cuestión fundamental son las disparidades en cuanto a derechos laborales, y por tanto ciudadanos, capacidades de articulación, objetivos y horizonte vital. Ciertamente que también aquí hay diferencias internas: entre quienes por origen social, estudios y sexo saben que esa situación setá transitoria -aquellos que por origen familiar provienen de las categorías socioeconómicas altas o intermedias - y quienes tienen bastantes probabilidades, por la otra cara de los mismos motivos, de que se alargue indefinidamente.

Ante la drástica disminución del empleo que la innovación tecnológica está provocando y la no superación del dualismo social que provoca el paro y que no se soluciona ni con subvención -en el supuesto que ésta se diera, que no es el caso- porque estamos hablando de un paro estructural, no parece haber otra salida progresista que el reparto del empleo, redefiniendo, desde ese reparto, el concepto del pleno empleo. La Enquesta no ha entrado en el análisis de estrategias de los diversos sujetos sociales, pero sí aporta aquí un dato muy significativo, el de la jornada laboral.

Con la incidencia que tienen el paro y el trabajo precario y sumergido, con un 10 por ciento de la población que trabaja menos de 20 horas por semana, resulta que el 42 por ciento de los ocupados están trabajando más de 40 horas semanales (el 15 por ciento más de 50 ). Se podría pensar que son los bajos salarios los que empujan a realizar horas extras y segundos trabajos; los datos nos demuestran que no es así, puesto que este fenómeno está mucho más extendido en las categorías altas (profesionales, gerentes, técnicos altos) con un 66 por ciento que trabajan más de la jornada legal y entre las medias (técnicos medios, empleados y pequeños empresarios) con un 44 por ciento, que entre las bajas (obreros y trabajadores de los servicios) que sólo están en un 32 por ciento.

En resumen, la posición ante el trabajo, en la que, a su vez, influye 
el nivel de estudios, la edad y el sexo y el propio origen social, parece ser el eje en torno al cual giran nuevas estructuraciones y que provoca desestructuraciones en la organización social tradicional.

\section{ESTRUCTURA SOCIAL Y DESIGUALDAD}

Diversos estudios, tanto en nuestro país (Documentación social, 1984, Miguélez, López, 1988) como en otros países (Boyles, 1983) ponen de manifiesto que crece la proporción de familias pobres, es decir, aquellas que no llegan a un nivel aceptable de ingresos en un país o región detetminada (según criterio bastante extendido, este nivel aceptable se establecería en la mitad de la tenta media). Hay una cierta coincidencia en calificar este fenómeno como de «nueva pobreza» para significar que se trata de personas o familias que anteriormente - cuando eran activos o cuando trabajaban o cuando trabajaban en «trabajos buenos»- estaban en mejor situación. En otras palabras: se trata de un tipo de pobreza que tiene que ver con los cambios en el trabajo a los que hemos aludido anteriormente o con una insuficiente intervención del Estado en favor de quienes ya han salido del mercado de trabajo.

Asimismo se refuerza la hipótesis, aunque de ello hay menos evidencia empírica, que los sectores altos son cada vez más ticos; también esto condice con el desatrollo de las nuevas capas medias y de las «nuevas elites» a las que nos referiamos, sin olvidar, por supuesto, la permanencia de las viejas elites y su capacidad de controlar recursos. Es decir, la reestructuración social parece ir acompañada de un aumento de las desigualdades en los países desarrollados.

Para el Átea de Barcelona estas hipótesis patecen confirmarse plenamente. Así, mientras una tercera parte de las familias de categorias bajas están en situación de pobreza, tal cosa acaece sólo a un 5,4 por ciento de las familias de categorías altas y a un 15 por ciento de las familias de categotías medias (en el primer caso están principalmente jubilados y en el segundo jubilados y trabajadores autónomos). Proporcionalmente, mayores son las distancias por lo que se tefiete al bienestar: un 14,2 por ciento de las familias de categorías altas son acomodadas, frente a un 1 por ciento de las categorias bajas y a un 4 por ciento de las categorias medias. Por tanto, se configura una franja de privilegio que prácticamente está centrada en el segmento alto de las categorías altas, frente a otra franja de marginación que principalmente está en las bajas y en los sectores inactivos de las medias. 
No tenemos datos de años anteriores que nos permitan evaluar la tendencia del fenómeno, pero sí algunas pistas que hacen suponer que las desigualdades pueden acrecentarse. En efecto, siendo el origen de la unueva pobrezar la situación tespecto al trabajo o respecto a la política social (jubilaciones, pensiones) cabe pensar que tamto la precarización del empleo y el paro como el debilitamiento del Estado del Bienestar son factores que presionan hacia el aumento de la nueva pobreza. Igualmente, y desde el otro lado, cabría evaluar los importantes aumentos de ingresos que se están registrando en ciertos sectores de técnicos y profesionales.

Pero la complejidad del sistema de desigualdad hoy lo percibimos mejor si tenemos en cuenta que las difetencias no tienen lugar sólo en el ámbito de los recutsos materiales, sino también en el que podríamos Hlamar sociocultural. El acceso a la educación, las actividades culturales, ,el uso del tiempo libre, la movilidad en el tertitorio y un largo etcéteta marcan distancias importantes entre el grupo que hemos llamado de pobres y el que hemos calificado de acomodados. Por ilustrat sólo uno de esos aspectos: los sectores pobres tienden a tener un ocio dominado pot la pasividad (ver TV), los acomodados un acio en el que la actividad tiene un importante protagonismo (lectura, viajes, actividades culturales); en un mundo tan rápidamente cambiante en el que la información, los conocimientos, las relaciones juegan un papel cada vez más clave, no es sólo cuestión de usar el tiempo de una u otra forma, sino de que usándolo de una manera o de otra se potencian las oportunidades de cara al futuro o se retrocede en ese terteno. En resumen, las distancias seríaladas podrían ser definidas en términos de autonomía, actividad, dominio, control e independencia para los sectores más altos y en los términos contrarios para los sectores bajos.

Pero si estas desigualdades socioculturales $-y$ también las economicasm- están fuettemente marcadas por las distancias de clase, aunque clatamente tocadas por la desestructuración de clases, otros factores influyen $y$ es recesario que los tengamos en cuenta, en particular el sexo y la edad; las mujeres, en particular cuando viven solas o son cabezas de familia, los viejos y los jóvenes se encuentran en una posición claramente infetior a los adultos varones. En algunos casos - los jóvenes o las mujeres casadas-- la estructura familiar los «salva» de la matginación, peto a costa "de acentuat o consolidar las «desigualdades interiores» a nivel micro, cuestión que no conviene olvidar si quetemos razonar en términos de igualdad y de dignidad de todas las personas. 


\section{¿HACIA UNA SOCIEDAD DUAL INTEGRADA?}

Los datos que arroja la Enquesta Metropolitana de Barcelona refuerzan, a mi entender, una interpretación de la relación estructura de clases desigualdad en términos de sociedad que se dualiza.

La nueva estructuración de clases artoja hacia sus márgenes a colectivos muy importantes de la antigua clase obrera y de las viejas capas medias: a los parados de larga duración que, con seguridad, ya no van a encontrar un trabajo estable ni una ocupación que les dé un estatus estable; a quienes tienen trabajos precarios o «trabajos malos», como los califican algunos estudiosos (Giovannini, 1989; Accorneto, 1985); a gran parte de los jubilados que no pueden mantener las condiciones de vida que tenían; a trabajadores autónomos que saltan de uno a otro «negocio» sin capacidad de asentamiento:

Estos sectores tienen una característica fundamental, que es el estancamiento, en un mundo que cambia con una rapidez frenética y ello los lleva tendencialmente hacia la marginalidad. No se trata sólo de que sus condiciones económicas sean muy débiles $\mathrm{y}$, aparentemente, cada vez más distantes de los niveles medios. La matginalidad es también política: aquí están las grandes bolsas abstencionistas, la falta total de asociacionismo, la desinformación aun para obtener recursos a los que tendrían derecho según la ley. $\mathrm{Y}$ es, asimismo, cultural, puesto que su uso del tiempo se caracteriza principalmente por la pasividad.

A pesar de ello no se barrunta, ni en nuestro país ni en los de nuestro entorno, una reacción de estos sectores contra su situación. Es más, la perspectiva que domina también en estos colectivos es la de creer en un futuro mejor para sí mistnos o para los hijos, principalmente a través de la educación superior de éstos. Es la imagen de la integración, pero una integración pasiva, silenciosa y subordinada.

Esta sociedad dual, profundamente injusta y desequilibrada, por más que a menudo la basura se guarde en el patio de atrás -la gente se avergüenza de ser pobre- es una sociedad preñada de conflictos y de insatisfacción que los políticos y los estudiosos harian bien en no olvidar. 


\section{REFERENCIAS BIBLIOGRAFICAS}

A. Accornero: I paradossi della disocupazione, Bologna, Il Mulino, 1986.

R. Boyles: Informe sobre la pobreza en la Comunidad Europea, Parlamento Eutopeo, 1983.

Documentación Social: «Pobreza y marginación en España», núm. 56, Madrid, 1984.

A. Giddens: La estructura de clases en las sociedades avanzadas, Madrid, Alianza, 1979.

P. Giovannini: «Sindicatos y telaciones laborales en Italia», Papers, núm. 32, Barcelona, 1989.

Mc Lennan y otros: State and Society in Contemporary Britain, Cambridge, Polity Press, 1984.

F. Miguélez y P. López: La pobresa a l'Àrea.

Metropolitana de Barcelona, Barcelona, Institut d'Estudis Metropolitans, 1988.

F. Miguélez y C. Solé: Classes socials i poder politic a Catalunya, Barcelona, PPU, 1987.

M. Piore y L. Toltaria: El mercado de trabajo. Teorias y aplicaciones, Madrid, Alianza, 1983.

Edwars, Gordon, Reich: Trabajo segmentado, trabajadores divididos, Madrid, Ministerio de Trabajo, 1985. 